Konya Mühendislik Bilimleri Dergisi., c. 8, Özel Sayı, ss. 9-14, 2020

Konya Journal of Engineering Sciences v. 8, Special Issue, pp. 9-14, 2020

ISSN: 2667-8055 (Elektronik)

DOI: $10.36306 /$ konjes.822187

\title{
DÜŞÜK MALIYYETLİ SÜREKLİ DALGA DOPPLER RADARI İLE TEMASSIZ YAŞAMSAL BELİRTİ ÖLÇÜMÜ
}

\author{
1'̈brahim ŞEFLEK ${ }^{\text {iD }}{ }^{2}$ Ercan YALDIZ ${ }^{\text {iD }}$ \\ 1,2Konya Teknik Üniversitesi, Mühendislik ve Doğa Bilimleri Fakültesi, Elektrik-Elektronik Mühendisliği Bölümü, \\ Konya, TÜRKIYE \\ ${ }^{1}$ iseflek@ktun.edu.tr, ${ }^{2}$ eyaldiz@ktun.edu.tr
}

(Geliş/Received: 05.11.2020; Kabul/Accepted in Revised Form: 01.12.2020)

ÖZ: Hayati sinyallerin temassız olarak uzaktan algılanması birçok uygulama açısından önem arz etmektedir. Bu algılamayı gerçekleştiren radarlar biyoradar olarak adlandırılmaktadır. Biyoradar kişinin solunum ve kalp atışından kaynaklanan göğüs duvarı hareketinin değişimiyle Doppler prensibini kullanarak hayati sinyallerin doğru bir şekilde ölçülmesini sağlamaktadır. Bu çalışmada, $24 \mathrm{GHz}$ çalışma frekansına sahip düşük maliyetli sürekli dalga (CW) Doppler radarı kullanılarak insan denekten temassız bir şekilde yaşamsal belirti (solunum, kalp atış hızı) ölçümleri gerçekleştirilmiştir. Ölçümlerden elde edilen sinyallerin işlenmesinde iki farklı yöntem kullanılmıştır. İlk yöntem Hızlı Fourier Dönüşümünü (FFT) esas alırken ikinci yöntemde Dalgacık yöntemine dayalı Çoklu Çözünürlük Analizi (MRA) yöntemi kullanılmaktadır. Solunum hızında birinci ve ikinci yöntem için elde edilen sonuçlar $\% 3.75$ ve $\% 0^{\prime}$ hata oranlıdır. Kalp atışı için sırasıyla \%9.35 ve \%8.45 hata oranlı değerler elde edilmiştir. Bu sonuçlar özellikle radarların tıbbi uygulamalar için başarıyla kullanılabileceğini göstermektedir.

Anahtar Kelimeler: Doppler radar, Radar sinyal işleme, Hayati sinyal tespiti, Temassız ölçüm, Biyoradar

\section{Contactless Vital Signs Measurement with Low Cost Continuous Wave Doppler Radar}

ABSTRACT: Remote sensing of vital signals without contact is important for many applications. Radars that perform this detection are called bio-radar. Bio-radar provides accurate measurement of vital signals using the Doppler principle with the change of chest wall movement caused by a person's breathing and heartbeat. In this study, non-contact vital signs (respiration, heart rate) measurements for human subject were performed using a low cost continuous wave (CW) Doppler radar with a $24 \mathrm{GHz}$ operating frequency. Two different methods have been used to process the signals obtained from the measurements. While the first method is based on the Fast Fourier Transform (FFT), the second method uses the Multi-Resolution Analysis (MRA) method based on the Wavelet method. The results obtained by the first and second methods for respiration are $3.75 \%$ and $0 \%$ error rates, respectively. These values for heartbeat are $9.35 \%$ and $8.45 \%$. These results show that radars can be used successfully for medical applications.

Key Words: Doppler radar, Radar signal processing, Vital signs detection, Non-contact measurement, bio-radar

\section{GİRIŞ (INTRODUCTION)}

Nesnelerin uzaktan temassız algılanması yirminci yüzyılda özellikle savaşların seyrini değiştirecek öneme sahip olmuş ve radarların ne kadar önemli birer donanım olduğunu ortaya koymuştur. Askeri kullanım amacıyla ortaya çıktığı ilk dönemlerinin aksine radarlar bugün hemen hemen hayatın her alanında karşımıza çıkmaktadır. Elektronik devre üretim teknolojisinin gelişimi bu sonucu ortaya 
çıkarmış ve radar boyutları oldukça küçülmüştür (Azevedo ve McEwan 1997; Andersen ve diğ., 2017). Özellikle iç ortam kullanımları için uygun hale gelen radarlar, daha önce kablolu olarak gerçekleştirilen uygulamaların temassız olarak gerçekleştirme fikrini gündeme getirmiştir (Amin ve diğ. 2017). Bu durum tıp alanında da kendine yer bulmuştur. Farklı radar tipleriyle; yaşlı ve hasta gözetimi, yanık ve yeni doğan vakalarının yaşamsal belirti takibi ve uyku apnesi gibi durumlarda radarların kullanılabileceği öne sürülmüştür (Hu ve diğ., 2013; Anishchenko ve diğ., 2019; Lin ve diğ., 2016; Adib ve diğ.,2015; Qi ve diğ.,2016). Çalışmaların temelini insanın solunum ve kalp atışından kaynaklı göğüs kafesi hareketinin tespiti oluşturmaktadır. Böylece yaşamsal belirti sinyalleri elde edilebilmektedir (Islam ve diğ., 2020; Seflek ve diğ., 2020; Abdul-Atty ve diğ., 2020; Acar ve diğ., 2021). Radarlar sağlık çalışanlarının işini kolaylaştırmanın yanı sıra, salgın hastalık vb. durumlarda onların korunması için de oldukça önemlidir. Ayrıca kablo ve prob gibi hastayı rahatsız eden, hatta yanık vakalarında ekstra yaralanmalara sebep olan durumlardan da hastayı kurtarabilecektir. Bu çalışmada sağlıklı bir gönüllü insan denekten düşük maliyetli bir CW Doppler radar kullanılarak, uygun sinyal işleme yöntemleri ile yaşamsal sinyaller elde edilmiştir.

\section{MATERYAL VE YÖNTEM (MATERIAL AND METHOD)}

Bir CW Doppler radarı sabit frekansta ürettiği sinyali verici antenle hedefe gönderir. Gönderilen sinyal ifadesi,

$S_{T X}(t)=A_{T} \cos \left(2 \pi f_{c} t+\phi(t)\right)$

şeklinde gösterilir. Burada $A_{T}$ gönderilen sinyalin genliğini, $f_{c}$ gönderilen sinyalin frekansını ve $\phi(t)$ ise faz gürültüsünü göstermektedir. Hedeften yansiyan sinyal, hedef hareketi kaynaklı faz gecikmesiyle birlikte alıcı anten vasıtasıyla alınır. Alınan sinyal ifadesi,

$S_{R X}(t)=A_{R} \cos \left(2 \pi f_{c} t-\frac{4 \pi d_{0}}{\lambda}-\frac{4 \pi x(t)}{\lambda}-\phi\left(t-\frac{2 d(t)}{c}\right)\right)$

şeklinde olur. Elde edilen sinyalde $A_{R}$ alınan sinyalin genliği, $d_{\mathrm{o}}$ hedefin radardan olan uzaklığ ve $x(t)$ hedefin hareketidir. $\phi\left(t-\frac{2 d(t)}{c}\right)$ ifadesi gecikmiş faz gürültüsünü, $d(t)$; $d_{0}$ ve $x(t)$ uzaklıkları toplamını ve $\frac{2 d(t)}{c}$ ise radar ile hedef arasındaki uçuş süresini göstermektedir. Alınan sinyal bir karıştırıcı vasıtasıyla gönderilen sinyalle temel bant seviyesine indirgenir. Temel bant sinyali,

$\mathrm{B}(\mathrm{t})=\cos \left(\frac{4 \pi \mathrm{d}_{0}}{\lambda}+\frac{4 \pi \mathrm{x}(\mathrm{t})}{\lambda}+\Delta \phi(\mathrm{t})\right)$

olarak elde edilir. $\Delta \phi(t)=\phi(t)-\phi\left(t-\frac{2 \mathrm{~d}(\mathrm{t})}{c}\right)$ ifadesi rezidual faz gürültüsüdür. Eşitlik (3)'te görüleceği gibi, sinyal çıkışı radarın hedeften olan uzaklığına bağlıdır. $\left(4 \pi \mathrm{d}_{0}\right) / \lambda$ terimi faz sinyalinde DC ofsete sebep olmaktadır ve faz kayması sonucu algılama sonucunu etkilemektedir. $(4 \pi \mathrm{d} 0) / \lambda$ teriminin $\pi / 2$ 'nin tek ve çift katlarına bağlı olarak, ideal sonuç yani hedef hareketi ile uyumlu çıkış ve boş nokta (null point) hedef hareketi ile uyumsuz çıkış ürettiği görülmektedir. Bu sorunu ortadan kaldırmak ve daima ideal bir sonuç elde edilebilmesi için dördün (quadrature) alıcılı CW radar kullanılmıştır. Dördün alıcıda birbirinden $90^{\circ}$ faz farklı iki çıkış üretilmesi bu sorunu gidermektedir. Çıkışta kanalın biri daima ideal sonucu üretmektedir. Eşitlik (4) dördün alıcılı CW radarın temel bant çıkışlarını göstermektedir.

$\mathrm{B}_{\mathrm{I}}(\mathrm{t})=\mathrm{A}_{\mathrm{I}} \cos \left(\frac{4 \pi \mathrm{d}_{0}}{\lambda}+\frac{4 \pi \mathrm{x}(\mathrm{t})}{\lambda}+\Delta \phi(\mathrm{t})\right)$
$\mathrm{B}_{\mathrm{Q}}(\mathrm{t})=\mathrm{A}_{\mathrm{Q}} \sin \left(\frac{4 \pi \mathrm{d}_{0}}{\lambda}+\frac{4 \pi \mathrm{x}(\mathrm{t})}{\lambda}+\Delta \phi(\mathrm{t})\right)$ 
Kanalların arktanjant demodülasyonu ile birleştirilmesi, kanal seçimi işlemini ortadan kaldırmakta ve yüksek doğrulukta daha hızlı sonuçlar vermektedir. Arktanjant demodülasyonu sonucu,

$\left.\phi(t)=\arctan \left(\frac{B_{Q}(t)}{B_{I}(t)}\right)=\frac{4 \pi d_{0}}{\lambda}+\frac{4 \pi x(t)}{\lambda}+\Delta \phi(t)\right)$

ifadesi elde edilir. Böylece faz sinyali, hedef hareketi ile doğrudan orantılı hale gelmektedir. Yaşamsal belirti sinyalleri elde edilen bu faz sinyalinden ortaya çıkarılmaktadır.

\section{Ölçüm Düzeneği ve Ölçümlerin Gerçekleştirilmesi (Measurement Setup and Performing Measurements)}

Yaşamsal belirti ölçümlerinin gerçekleştirilmesinde RF-Beam firmasının ürettiği K-LC6 radar modülü kullanılmaktadır. Radar 24 GHz çalışma frekansı ve dördün alıcı yapısına sahiptir. Şekil-1 K-LC6'nın blok diyagraminı göstermektedir.

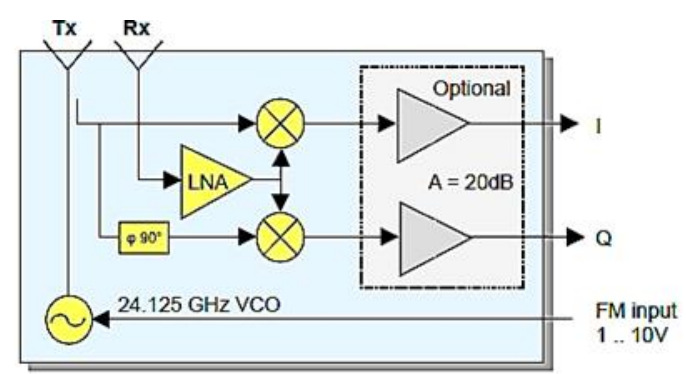

Şekil 1. K-LC6 blok diyagramı

Figure 1. Block diagram of K-LC6

Temel bant çıkış sinyalleri I ve Q, AD-620 mikrovolt sinyal modülü ile kuvvetlendirilmektedir. Solunum ve kalp atış sinyallerinin frekansı sırası ile 0.1-0.5 ve 0.8-2 Hz arasında değişmektedir. Kuvvetlendirilen sinyaller hem bu frekans bant aralıklarını kapsayacak şekilde hem de örtüşmeyi (aliasing) önlemek amacıyla gerçekleştirilen $30 \mathrm{~Hz}$ kesim frekanslı aktif alçak geçiren filtre ile filtrelenmektedir. Sinyaller işlenmesi için VTK 1050 veri toplama cihazı ile $200 \mathrm{~Hz}$ örnekleme frekansında sayısallaştırılarak bilgisayara kaydedilmektedir. Ölçüm için sağlıklı bir gönüllü denek radarın $1 \mathrm{~m}$ karşısına oturtulmaktadır. Sabit bir şekilde oturan denekten 60 saniye boyunca ölçümler alınmaktadır. Şekil-2 ölçüm ortamını göstermektedir.

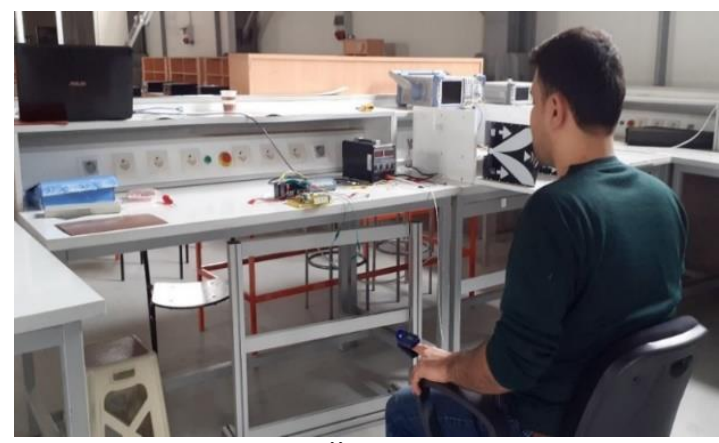

Şekil 2. Ölçüm ortamı

Figure 2. Measurement environment

\section{BULGULAR (RESULTS)}

Elde edilen radar sinyallerini işlemek için iki farklı yöntem kullanılmaktadır. Birinci yöntemde I ve $Q$ temel bant sinyallerine normalizasyon uygulanmaktadır. Ardından arktanjant demodülasyonu ile birleştirilen I ve $Q$ sinyallerinin faz bilgisine ulaşılmaktadır. Faz bilgisinin doğru bir şekilde elde edilmesi 
için, arktanjant demodüleli sinyale faz açma (unwrapping) işlemi uygulanır. Arktanjant fonksiyonu $(\pi / 2,-\pi / 2)$ aralığında değerler almaktadır. Hedef hareketi büyük olursa bu değerler aşılmakta ve faz sinyalinde süreksizlik meydana gelmektedir. Bu süreksizliklerin ortadan kaldırılabilmesi için sinyal işlemede $\pi^{\prime}$ nin tamsayı katları ile çarpılarak faz açma işlemi gerçekleştirilir. Faz sinyali, solunum sinyalini elde etmek için 0.1-0.5 Hz frekans aralığında bant geçiren filtre ile filtrelenmektedir. Benzer şekilde 0.8-2 $\mathrm{Hz}$ aralığında bant geçiren filtre kalp atış sinyalini elde etmek amacıyla faz sinyaline uygulanmaktadır. Sinyal periyodikliğini artırma amacıyla otokorelasyon gerçekleştirilmektedir. Son olarak sinyale Hızlı Fourier Dönüşümü (FFT) uygulanmaktadır. Solunum ve kalp atış hızı tespit edilmektedir. Şekil-3 sinyalin işlenmesi için kullanılan birinci yöntemin blok diyagramını göstermektedir.

Sinyallerin işlenmesi için kullanılan ikinci yöntemde ise sinyaller ilk yönteme benzer şekilde normalizasyona, arktanjant demodülasyonuna ve faz açma işlemine tabi tutulmaktadır. Demodüle edilmiş sinyale çoklu çözünürlük analizi (MRA) uygulanmaktadır (Mallat, 1989). MRA, sinyali yaklaşım (CA) ve detay katsayılarına (CD) ayırarak, sinyalin değişken çözünürlükteki ikili frekans bantlarında incelenmesini sağlamaktadır. İkili frekans bantları kullanılarak sinyal ayrışımı ve ilgilenilen fizyolojik sinyallerin çıkarılması sağlanmaktadır. MRA için ana dalgacık fonksiyonu olarak Symlet 7 kullanılmaktadır. Şekil-4 sinyalin işlenmesi için kullanılan ikinci yöntemin blok diyagramını göstermektedir.

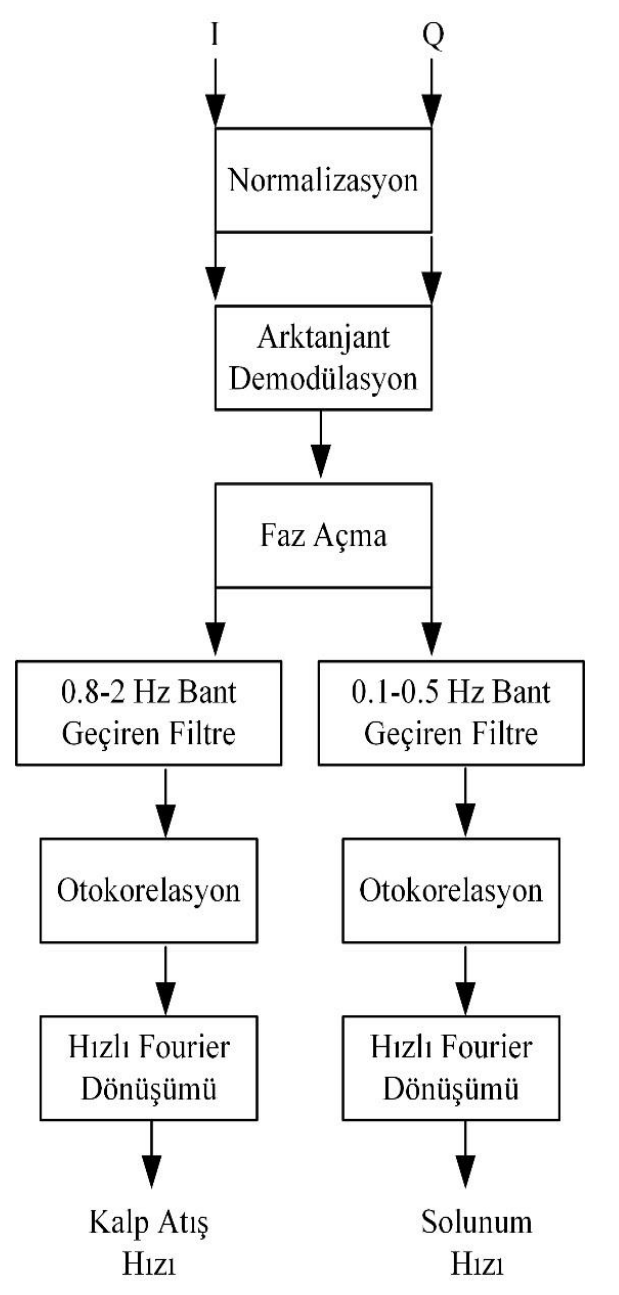

Şekil 3. Kullanılan birinci yöntemin blok diyagramı Figure 3. Block diagram of the first method used

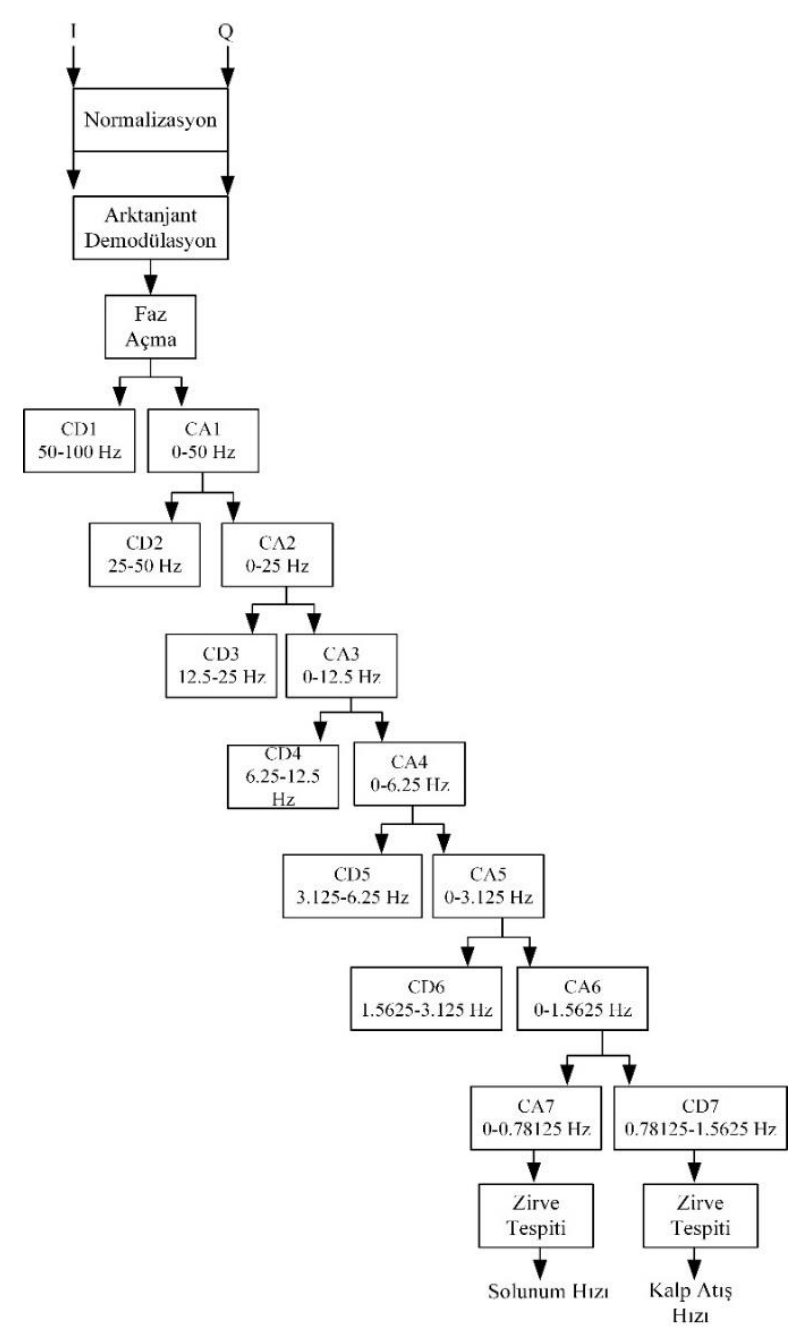

Şekil 4. Kullanılan ikinci yöntemin blok diyagramı Figure 4. Block diagram of the second method used

Birinci yöntem kullanılarak gerçekleştirilen bir ölçümün sonuçları Şekil-5'te gösterilmektedir. Şekil6’ da aynı ölçüm için ikinci yöntem kullanarak elde edilen sonuçlar gösterilmektedir. Ölçüm sonuçlarının 
doğruluğunun karşılaştırılması amacıyla referans olarak Veron 501 pulse oksimetresi kullanılır. Ayrıca solunum referansı için ölçüm sırasında nefes alış-verişi denek tarafından sayılmaktadır.

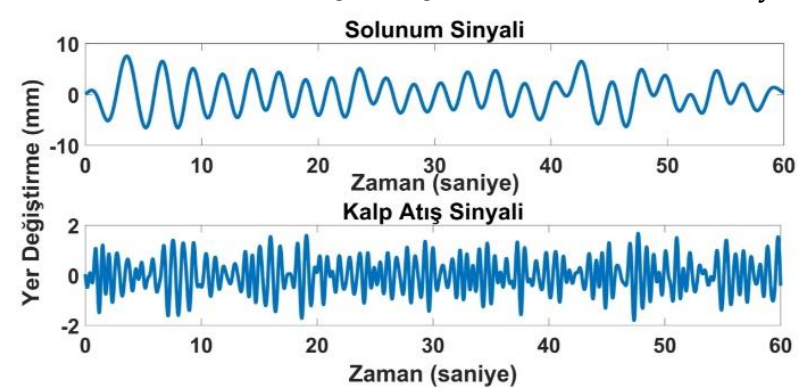

(a)

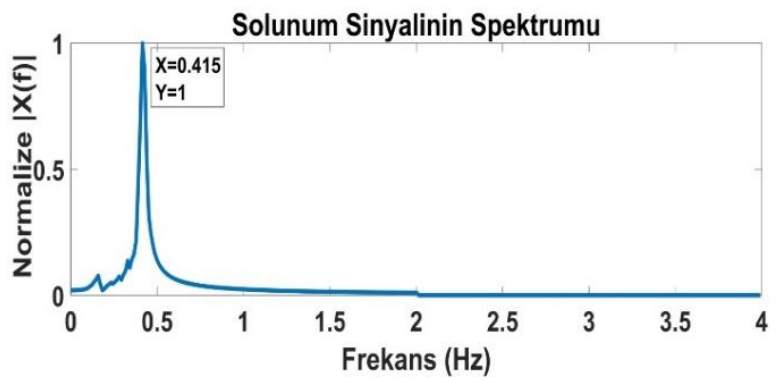

(b)

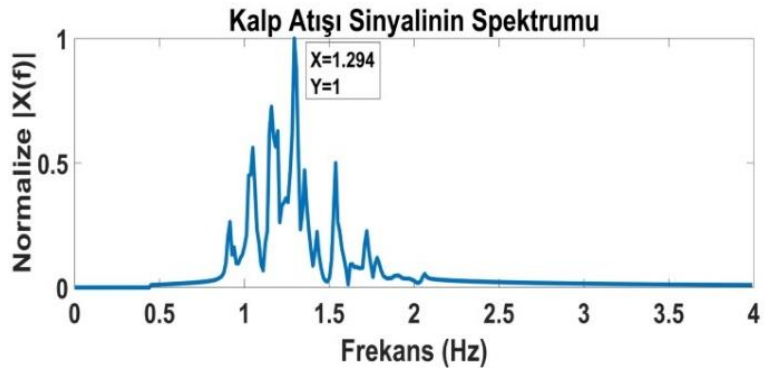

(c)

Şekil 5. Birinci yöntem kullanılarak elde edilen ölçüm sonuçları (a) Solunum ve kalp atış sinyali (b)

Solunum sinyalinin frekans spektrumu (c) Kalp atışı sinyalinin frekans spektrumu

Figure 5. Measurement results obtained using the first method (a) Respiration and heartbeat signal (b) Frequency spectrum of respiration signal (c) Frequency spectrum of heartbeat signal

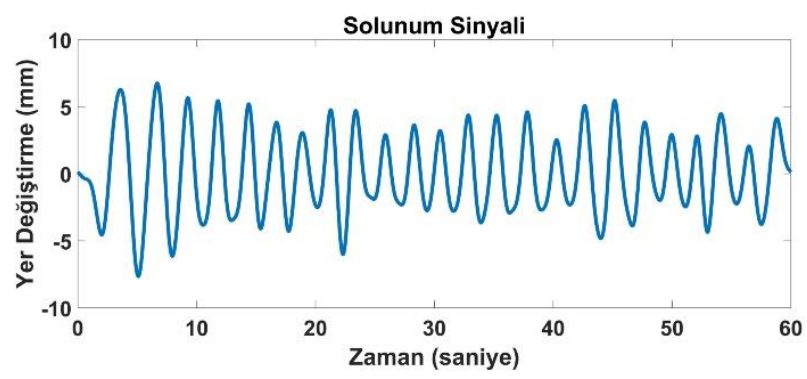

(a)

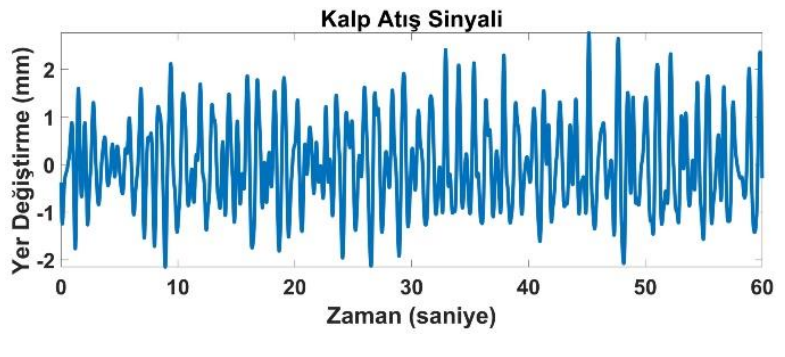

(b)

Şekil 6. İkinci yöntem kullanılarak elde edilen ölçüm sonuçları (a) Solunum sinyali (b) Kalp atış sinyali Figure 6. Measurement results obtained using the second method (a) Respiration signal (b) Heartbeat signal

İki yönteme ait ölçüm sonuçları, referans değerlerle karşılaştırılarak yüzde hata oranları ile sunulmaktadır. Solunum için referans değeri 24 tür. Birinci yöntemde bu değer \%3.75 hata ile 24.9 olarak hesaplanmıştır. İkinci yöntemde \%0 hata ile 24 solunum sayısı hesaplanmıştır. Kalp atış ölçümlerinde referans 71 atımdır. İki farklı yöntem için sırasıyla \%9.35 hata ile 77.64 ve \%8.45 hata ile 65 değerleri 
bulunmuştur. Sonuçlar incelendiğinde çoklu çözünürlük analizinin (MRA) kullanıldığı yöntemin daha başarılı olduğu görülmektedir.

\section{SONUÇLAR (CONCLUSIONS)}

Düşük maliyetli $24 \mathrm{GHz}$ çalışma frekansında çalışan bir CW Doppler radarı kullanılarak sağlıklı bir bireyin temassız yaşamsal belirti ölçümleri gerçekleştirilmiştir. Ölçüm sonucu elde edilen sinyallerin işlenmesinde iki farklı sinyal işleme yöntemi kullanılmıştır. Kullanılan yöntemlerin hata oranı solunum sinyali tespiti için $\% 3.75$ ve $\% 0$ olmuştur. Kalp atış sinyali için bu oranlar $\% 9.35$ ve $\% 8.45$ 'tür. Sonuçlardan MRA yönteminin daha başarılı olduğu görülmektedir. Kalp atış sinyalinin hata oranının yüksek olması kalbin çok küçük yer değiştirme hareketinden kaynaklandığı değerlendirilmektedir. Bu sonuçlar radarların temassız ölçümlerde sağlık çalışanı ve hastalara sağladığı avantajlarla tıp alanında gelecekte yoğun bir şekilde kullanılabileceğini göstermektedir.

\section{KAYNAKLAR (REFERENCES)}

Abdul-Atty, M.M., Amar, A.S.I. ve Mabrouk, M., 2020, “C-Band FMCW Radar Design and Implementation for Breathing Rate Estimation", Advances in Science, Technology and Engineering Systems Journal, cilt. 5, no. 5, ss. 1299-1307.

Acar, Y. E., Saritas, I. ve Yaldiz, E., 2021, “An Experimental Study: Detecting the Respiration Rates of Multiple Stationary Human Targets by Stepped Frequency Continuous Wave Radar", Measurement, cilt.167,108268.

Adib, F., Mao, H., Kabelac, Z., Katabi, D. ve Miller, R. C. “Smart homes that monitor breathing and heart rate", Proceedings of the 33rd Annual ACM Conference on Human Factors in Computing Systems, ss.837-846, 2015.

Amin, M., 2017, Radar for indoor monitoring: detection, classification, and assessment, CRC Press.

Andersen N., Granhaug, K., Michaelsen, J. A., Bagga, S., Hjortland, H. A., Knutsen, M. R., ve Wisland, D. T., 2017, "A 118-mW pulse-based radar SoC in 55-nm CMOS for non-contact human vital signs detection", IEEE Journal of Solid-State Circuits, cilt.52, no.12, ss.3421-3433.

Anishchenko, L., Zhuravlev, A. ve Chizh, M., 2019, "Fall detection using multiple bioradars and convolutional neural networks", Sensors, cilt.19 no.24, ss.5569.

Azevedo, S. ve McEwan, T. E., 1997,“Micropower impulse radar", IEEE Potentials, cilt.16 no.2, ss.15-20.

Hu, W., Zhao, Z., Wang, Y., Zhang, H. ve Lin, F., 2013, “Noncontact accurate measurement of cardiopulmonary activity using a compact quadrature Doppler radar sensor", IEEE Transactions on Biomedical Engineering, cilt.61 no.3, ss.725-735.

Islam, Shekh MM, Motoyama, N., Pacheco, S. ve Lubecke, V. M.. "Non-Contact Vital Signs Monitoring for Multiple Subjects Using a Millimeter Wave FMCW Automotive Radar ", IEEE/MTT-S International Microwave Symposium (IMS),ss.783-786, 2020.

K-LC6 Radar Modülü. https://www.rfbeam.ch/product?id=12, ziyaret tarihi: 14.08.2020.

Lin, F., Zhuang, Y., Song, C., Wang, A., Li, Y., Gu, C. ve Xu, W., 2016, “SleepSense: A noncontact and costeffective sleep monitoring system", IEEE Transactions on Biomedical Circuits and Systems, cilt.11 no.1, ss.189-202.

Mallat, SG., 1989, "A theory for multiresolution signal decomposition: the wavelet representation", IEEE Trans Pattern Anal Mach Intell, cilt.11 no.7 s.674-693.

Qi, F., Li, C., Wang, S., Zhang, H., Wang, J. ve Lu, G., 2016, “Contact-free detection of obstructive sleep apnea based on wavelet information entropy spectrum using bio-radar", Entropy, cilt.18 no.8, ss.306.

Seflek, I., Acar, Y. E. ve Yaldiz, E., 2020, “Small Motion Detection and Non-Contact Vital Signs Monitoring with Continuous Wave Doppler Radars", Elektronika ir Elektrotechnika, cilt.26 no.3, ss.54-60. 